\title{
Designing an advanced software tool for Digital Scholarly Editions
}

\author{
The inception and development of EVT \\ (Edition Visualization Technology)
}

\author{
Roberto Rosselli Del Turco
}

\begin{abstract}
The increasing dissemination of Digital Scholarly Editions has highlighted not only the great potential of this method of publication, but also a good number of theoretical problems that affect both the DSEs as editorial products, and the impact of tools and methods of computer science on the methodology of textual criticism. On the one hand, the editions published so far are an evolution of the practice of ecdotics and represent not only a collection of interesting experiments, but also innovative and effective research tools. On the other hand, however, the limits within which an author of digital editions is forced to operate and the most appropriate strategies to minimize their impact have not yet been thoroughly investigated. The adoption of IT tools and methods, in fact, provides many answers to the requests of digital philologists, but the very nature of these tools imposes very strict modes of action, sometimes perceived as too rigid by the scholar. This article presents and describes a software tool that comes at the end of the process of creating a digital edition, to be used in that crucial phase when the edition is prepared for publication on the Web. The aim is not to show the more technical aspects of this tool, even if its fundamental characteristics will be introduced to better understand the terms of the issue, but to describe its genesis and development, and to highlight how visualization software represents a crucial element of the whole editorial process.
\end{abstract}

\section{Introduction}

$\mathrm{T}$ his article introduces EVT (Edition Visualization Technology), a software tool born in the context of an ongoing digital edition project, the Digital Vercelli Book (Rosselli Del Turco 2017, from now 
on DVB). ${ }^{1}$ This project, started about 2003 and now very close to conclusion, aims at creating a digital edition of the texts contained in the Codex Vercellensis (Vercelli, Archivio e Biblioteca Capitolare, MS CXVII), a particularly important manuscript since it is one of the few existing codices preserving poems composed in the Old English language. The researchers of the project team have encoded all texts according to the the TEI XML schemas (TEI Consortium 2019), the de facto standard with regard to semantic annotation of literary texts, so that it is possible to fully exploit the digital medium and offer valuable research and didactic tools: two edition levels (diplomatic and normalized transcriptions), text-image linking, hot-spots with details for specific manuscript areas, visualization of the manuscript quire structure, a powerful search engine, and more (Fig. 1).

The reasons why we started to explore the possibility of developing a software tool specifically for this project are many. When the encoding of all the manuscript texts neared completion and we started an inquiry on the state of the art with regard to edition browsing software (about 2010), the available tools were either proprietary (therefore available on a per license basis only, possibly with permission to make limited changes), or closely linked to a specific software platform (e.g., a given operating system, or even a specific version of a browser running on that operating system), or too complex to install and use (such as XML native databases), or, finally, tied to a specific project to such an extent that adapting them for our purposes would have required too much time and resources. Actually, several programs that we took into consideration suffered from two or more of these flaws. In spite of the sensible "do not re-invent the wheel" saying, we had to do just that. ${ }^{2}$

As a consequence, it has been our intention, from the very beginning, to develop a tool that would respect some basic requirements:

1. Project home page: http://evt.labcd.unipi.it/. For more details about EVT 1 see Rosselli Del Turco 2015. The reasons leading to the completely new EVT 2 version are discussed in Di Pietro - Rosselli Del Turco 2018.

2. Note that at the moment of writing the present article many more digital editions have been published, see Franzin 2016 and SA HLE 2017 for a quite comprehensive list. The available browsing tools are correspondingly more numerous and more powerful: see f.i. TEI Publisher (http://teipublisher.com/index.html), M. Burghart's TEI Critical Apparatus Toolbox (http://ciham-digital.huma-num. fr/teicat/index.php), The Versioning Machine 5.0 (http://v-machine.org/), and more. 


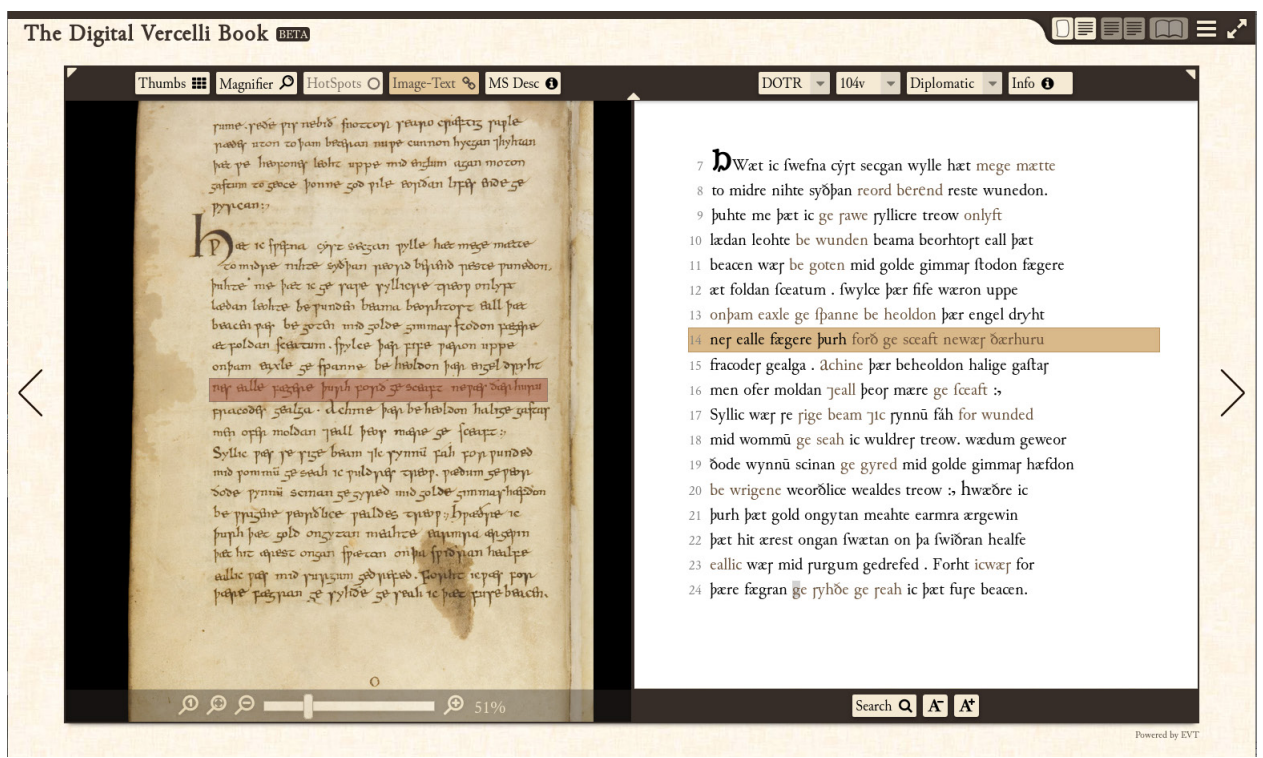

Figure 1. EVT 1: The Digital Vercelli Book

- the program was to be distributed as open source software, to make it freely available for the academic community;

- it had to be based on the major Web standards (hence the use of HTML, CSS, JavaScript, etc.) $)^{3}$ so that it would naturally be multiplatform and independent of specific software environments;

- it was going to be designed as a user-friendly tool both for the project editor, who should be able to publish the edition on a Web site without any server software being required, and the final user;

- finally, it was going to be highly flexible, to be used as a general purpose tool in other digital edition projects, possibly very different with regard to text language, literary period, genre, etc.

This criterion of flexibility proved to be fundamental as soon as researchers from other projects contacted us asking permission ${ }^{4}$ to use EVT to pub-

3. Since so many of the first pioneering wave of DSEs became unusable just a few years after their publication, we decided that a standard-compliant Web application would be the best solution to avoid the problem of technical obsolescence.

4. EVT is distributed as open source software, therefore it is available for anyone to use and/or modify as they see fit. While this may be an unnegotiated process, it is usually a better choice, and more productive in the long term, to engage with 
lish their digital editions. Requests for modification of existing features, and implementation of new features hitherto not taken into account because they were not useful or relevant for the DVB, have been the main source of innovation and evolution of this tool. One of the most basic features of diplomatic editions, for instance, is the presence of a document summary (regesto) for each published document: this feature had not been considered for inclusion in the DVB, since all the texts preserved in this manuscript are literary works, therefore it was necessary to implement this feature ex novo for the Codice Pelavicino Digitale project (Salvatori 2014, from now on CPD: Fig. 2). On the other hand, since the regesto is encoded in a TEI $<$ front> element, this allowed us to expand on the work done for the CPD and to turn it into a general feature. As a result, all editions needing an introduction for a specific text can now include it inside the $<$ front $>$, and have it conveniently displayed in the text frame. ${ }^{5}$

The exchange of ideas and views with other scholars engaged in the preparation of editions of different kinds of works has helped us enormously in the development of EVT, resulting in a much better product. ${ }^{6}$

The quest for flexibility, however, soon met the limits of the initial programming approach: EVT 1, which is still developed and maintained for the purpose of diplomatic and single-witness (codex unicus) critical editions, is based on an XSLT 2.0 transformation chain that takes as its input one or more XML documents in the standard TEI P5 format, which are turned into a Web-based application - a mix of HTML5, CSS3 and JavaScript - that can be easily shared on the Web. Unfortunately, with time the XSLT code-base has grown considerably and it is now quite complicated

the development team of any open source software and discuss about possible changes, new features, bug fixing, etc.

5. A similar process has taken place with regard to support for named entities, for lists and for the highlighting of specific markup elements: again, it was a request coming from the CPD, and again all projects that have this need can now satisfy it, possibly for very different purposes than those envisioned for the CPD.

6. Currently EVT is being used for several DSE publishing projects: besides the already mentioned VBD and CPD, see f.i. TARSIAN: A Digital Edition of Arabic Manuscripts of the Letters of Paul of Tarsus (http://tarsian.vital-it.ch/about/), UPenn Libraries LJS 225 - Liber Simulationis Litterarum (http://sceti.library.upenn. edu/ljs225/), the digital edition of Marciana Gr. Z 11 (379) (http://humarecviewer.vital-it.ch/), the digital edition of the Storia d'Italia by F. Guicciardini (http://triangle.ens-lyon.fr/spip.php?article5109), and The Marianne Moore Digital Archive (https://moorearchive.org/); more are in preparation, some of them making use of the development version (EVT 2, see below). 


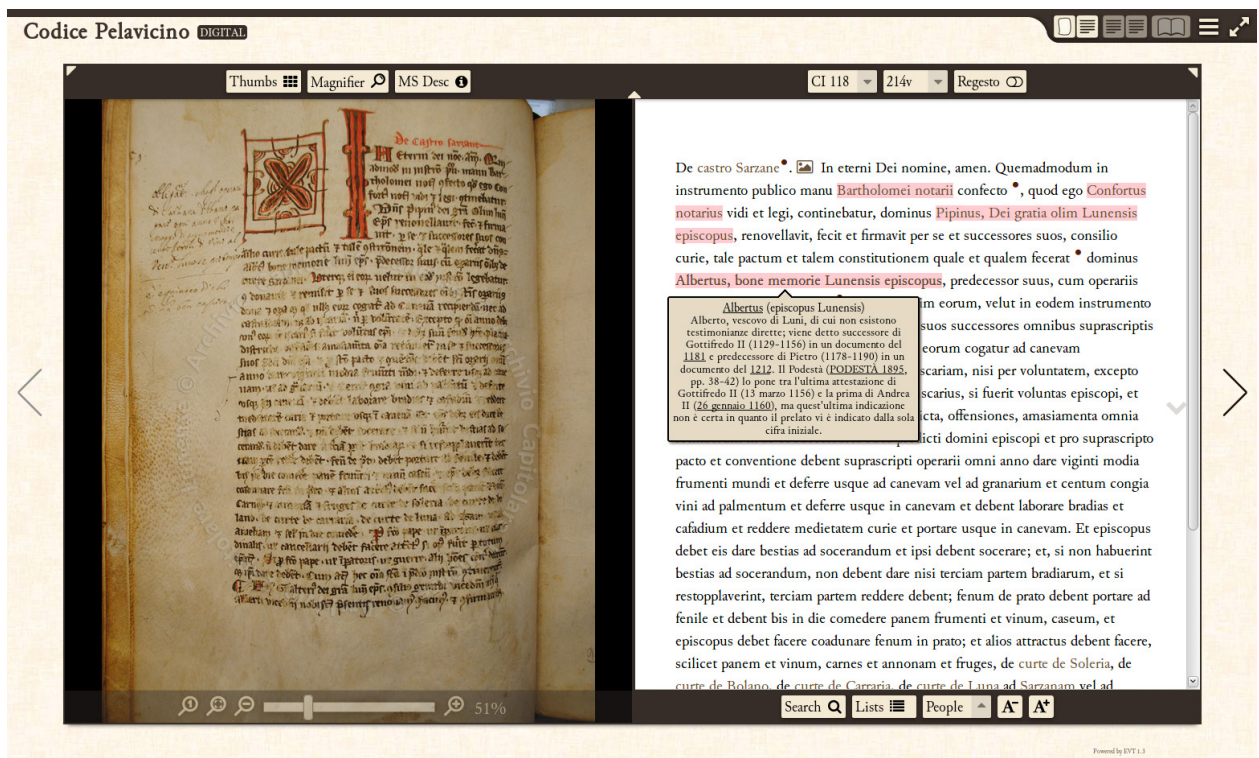

Figure 2. EVT 1: The Codice Pelavicino Digitale

to understand and modify, especially for a newcomer to the development team. Furthermore, the increasing number of available GUI (Graphical User Interface) components has made the general layout quite complex with regard to the interaction between them, and tricky to handle at the programming level. We needed more flexibility and modularity to make the development of new features easier and faster, and this has led to a completely new infrastructure.

\section{EVT 2.0}

One of the new features to be implemented, perhaps the most important one since it was both critical to the original project (the Digital Vercelli Book) and requested by many other scholars, was support for complex, multi-witness critical editions, again encoded according to the TEI schemas and Guidelines. As stated in the previous section, we quickly realized that the old programming framework, especially with regard to the code handling the UI (User Interface) layout, was woefully unfit for the task: there was no way that we could add such complex functionality to the existing code-base while adding a new layer of GUI objects to the Web application interface. 
Therefore we redesigned the whole application from scratch, adopting a completely new programming framework, and started developing the new complex feature of multi-witness critical edition support, with the ultimate goal of gradually bringing all the features of EVT 1 closely related to diplomatic and single-witness editions into the new version (Fig. 3).?

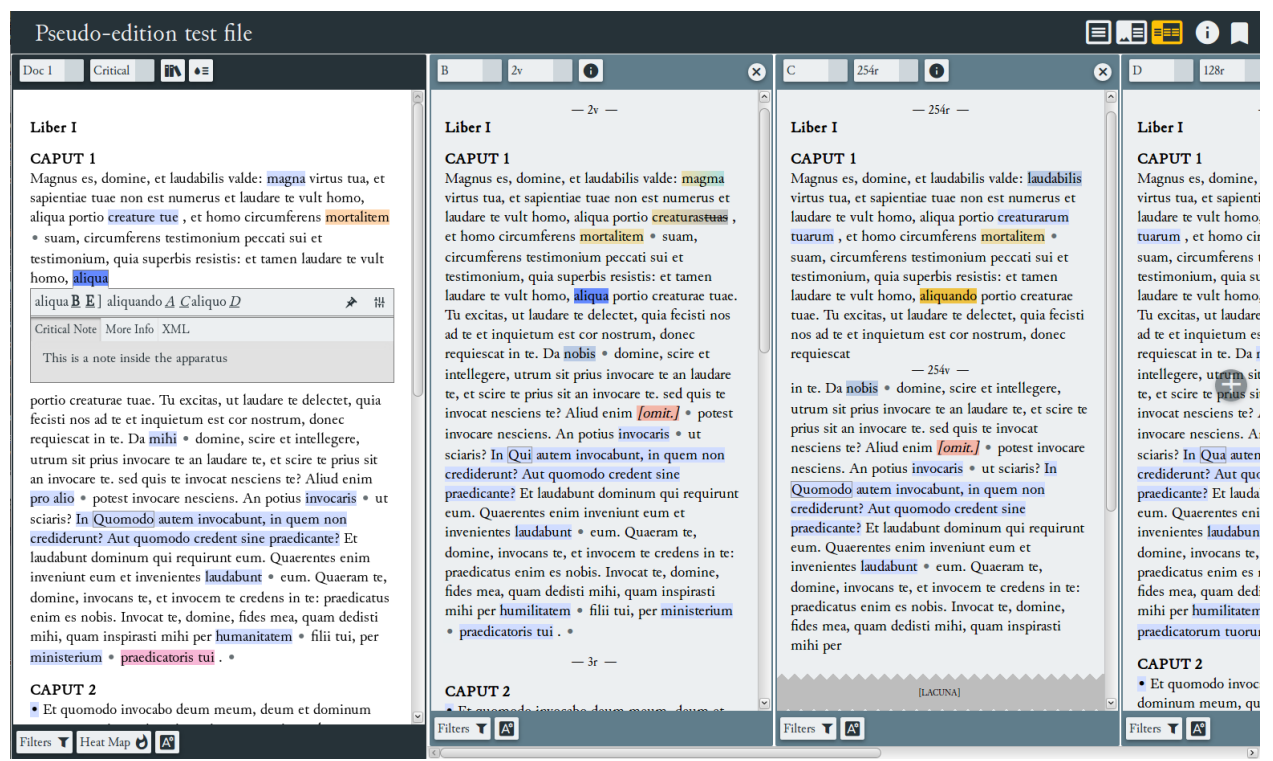

Figure 3. The new User Interface of EVT 2 (early alpha version)

We decided to abandon the XSLT transformation chain in favor of a set of JavaScript parsers specifically written to retrieve edition content directly from the XML document(s), to be accessed on the fly by means of an AJAX request. ${ }^{8}$ To develop the new version of EVT we decided to use AngularJS (https://angularjs.org/), a JavaScript framework specially designed for Single Page Applications. AngularJS implements the MVC (Model View

7. Since redesigning the whole application and bringing all the old features into it would have take a long time, we decided to continue the parallel development of new (but not too complex) functionalities in EVT1, at least until a stable and feature-complete version of EVT2 is implemented.

8. Asynchronous JavaScript and XML, a method to develop Web applications. See especially Garrett [2005] 2007. 
Controller) design pattern and therefore allows to separate the logic of data presentation from the core of their processing, providing great modularity.

Once the JavaScript parsers have retrieved the edition data, everything is saved in a temporary $\mathrm{JSON}^{9}$ structure, which persists until the Web application remains open and is organized in such a way that it can be easily and rapidly accessed when needed. The final styling of the digital edition is entrusted to CSS style-sheets and is easily customizable: the editor can add new CSS style rules in order to customize all aspects of text visualization, according to their needs. Modifying the existing CSS stylesheets is always an option, of course. ${ }^{10}$

This first version already provides good support for critical editions:

- the apparatus entries are displayed inline after clicking on the highlighted words in the text;

- both positive and negative apparatuses are supported, as well as critical notes inside an entry;

- witnesses are saved in a separate list and can be described in detail using the TEI $<$ msDesc $>$ element;

- the critical apparatus is a dynamic device, each witness siglum is a hyperlink that can lead to opening the text of that specific witness in the collation view;

- all witnesses' texts are generated automatically by the software and can be showed side by side with the critical text in the collation view;

- variants can be categorized and visualization filters applied (e.g., to only show substantive or orthographic variants);

- the current browsing position can be saved as a bookmark to be shared.

9. JavaScript Object Notation, a textual data format commonly used in browserserver communication. Official Web site: http://www.json.org/.

10. The customization of generic and linear TEI element is very simple, even if EVT does not yet consider them in the default visualization: in fact, the TEI elements which are not handled in any particular way by EVT are always transformed in HTML elements with the TEI tag name as class name. In this way, the customization is very easy: you only have to add a rule that matches the tag name of the TEI element to style. F.i., a deletion encoded with $<$ del $>$, can be displayed with a line through the deleted text just by adding the rule .del \{text-decoration: line-through;\}. 
From the point of view of the editor, the new architecture is as easy to use as the previous one: the only technical skill required from the editors is a general competence in XML editing to configure EVT properly and the care to place each XML-related component of the edition (mainly the schema besides the encoded texts) into the correct area of the directory structure.

One important aspect of the new infrastructure is that, even if the current JavaScript parsers can only process TEI XML documents, EVT has been designed to handle editions that are not encoded in this particular format. In fact, everything relies on the JSON structure mentioned above and to add the support for different formats (e.g. non-TEI XML, LaTeX, etc.) it is only necessary to implement new dedicated parsers that extract the edition data and pour them into the temporary JSON collections.

\section{Inspiring principles}

The design of EVT is based on two general principles that act as a cornerstone to all versions, present and future. The first one is the distinction between two types of users: the editor, the scholar responsible for the preparation and publication of the digital edition, and the reader, the final user of the latter. The primary objective for the editor is to enable him or her to prepare the edition according to the chosen ecdotic methodology for that specific edition project. Therefore, the inspiring principle, defined even before evaluating the possible technical solutions regarding the creation of this software, is absolute transparency regarding the work of the philologist, avoiding possible conflicts with the correct application of editorial practice: the validity from the philological point of view of the final result of the publishing process is the sine qua non of any digital project, just like for others of a more traditional type. A consequence of this approach is the search for maximum flexibility and configurability in relation to the functioning of the program. In fact, the possible combinations among methods of textual criticism (neo- and post-Lachmannism, "best text" edition, genetic criticism, new philology, documentary editions) and the nature of the works to be edited (language, cultural tradition, historical period, gender, etc.) are so numerous that, although it is impossible to predict every possible need, it is essential to offer the editor the possibility of configuring the final product, for example by choosing the methods of presentation of the texts, which tools to make available to the end user and the general layout of the digital edition. 
For both types of users, great care has also been taken with regard to user-friendliness. In the case of the editor, the choice to adopt the clientonly architecture means that installing the software is very simple (just unpack the archive on your hard disk), as well as deploying it to a Web server (the edition is a folder that can be copied to any server and is immediately usable by opening the index.html file) and the subsequent maintenance (virtually no maintenance is needed once the edition is up and running). As far as the end user is concerned, the user interface has been designed and tested in such a way as to ensure that EVT is an intuitive and easy-to-use tool. ${ }^{11}$

The second fundamental principle is the separation of edition data from the visualization tool: when building an edition by means of ad hoc software $^{12}$ very often a level of interconnection is established between the text of the edition and its navigation program in such a depth that it makes it very difficult to separate one from the other at a later date, with negative consequences not only as regards possible changes to be made to the published edition, but also for a possible reuse of textual data. EVT, on the contrary, clearly separates the two domains while remaining an easily customizable tool thanks to the rich configuration options: because of this approach it offers full support to the concept of interchange - if not interoperability — an almost impossible task for editions in which the data is either highly customized from the point of view of the TEI scheme, or are mixed in a variety of different formats (eg. RDF + TEI) that can be correctly interpreted only by that specific software, or again are stored in a relational database and extracted for display. The editor can therefore focus on preparing the edition encoding the texts using the TEI XML schemas, facilitated by the fact that we have endeavoured not to impose specific markup solutions for general encoding problems. As a further consequence

11. For a broader release, the development cycle starts with the creation of several UI mockups of the new and/or modified features, which are discussed within the team (design phase); after a consensus is reached about the best solutions to choose, development starts and a functioning prototype is built (implementation phase); the working prototype is proposed to external users for further testing, and adjustments are made on the basis of the feedback we receive (testing phase); when all the problems and bugs reported have been fixed the version is deemed to be ready for release.

12. Such editions are defined as haute couture in a metaphor coined by Elena Pierazzo (2016). To keep up with the metaphor, EVT aims at being a prêt-à-porter tool that allows very high quality, finely tailored results. 
of this choice, not only is it very easy to use the same data with more advanced and powerful versions of EVT, but if the latter is no longer considered suitable by the editor - for example because a better tool has been made available - he or she can migrate the data of an existing edition to the new and more advanced browsing software.

Finally, EVT's development model can be defined as "mixed". At first, considering the limited resources available for this initiative, EVT has been more of a project-based learning initiative at the University of Pisa (Digital Humanities degree course $)^{13}$ than anything else. In fact, there have been two abandoned iterations before the first usable version, employed to publish a first preliminary edition of the Vercelli Book in January 2014, was developed. We discovered that this was an impossible task for a single student, in fact things started to improve significantly only when a development team was created. At that point we managed to create a pool of knowledge, based on shared resources and communication tools, and what's more the development cycle turned out to be at least partly self-sufficient, with students tutoring other students.

Collaboration with other digital edition projects and funding for the development of specific features is the second method of development, the one that has allowed us to achieve the greatest progress. Note that these features will then be available to all users of the software, so that any improvement required by an EVT-based project is potentially useful and certainly applicable to all others who use it.

\section{Recent and future developments}

The first instance of the new version has been released in July 2016 (Di Pietro 2016), a more advanced beta version, with support for a fuller set of critical edition features, has been released in October 2017 (MARTiGNANO 2017) and as the previous one is available on the project home page (full announcement on the development blog (http://visualizationtechnology.wordpress.com/). To produce the latter version, the EVT development team has been working together with the researchers and scholars involved in the PhiBor - Philosophy on the Border of Civilizations project, in fact the default example used in the beta version (see Fig. 4) is based on a sample

13. I am very grateful to the founders and organizers of this degree course for training students well versed in both computer science and humanities. This has allowed a fruitful dialogue for both sides. 
prepared as a test for their forthcoming digital edition of Aristotle's Metaphysics as translated by Avicenna. As it was the case for the Codice Pelavicino Digitale, close cooperation with a complex and rich digital edition project has proved to be essential to refine and improve the set of features that EVT can offer to the academic community.

Besides the features introduced in the first, experimental version, the October 2017 version significantly improves the support for critical editions:

- in the reading view the critical apparatus has been moved in an independent panel, next to the text, the inline display is still used in the collation view;

- support for sources and analogues has been added to the aforementioned panel, text fragments are linked to the critical text;

- apparatus entries can be split into two or more groups of readings, depending on the witness families or other organizing criteria;

- the "Pin frame" area has been improved;

- support for textual traditions with multiple recensions has been added.

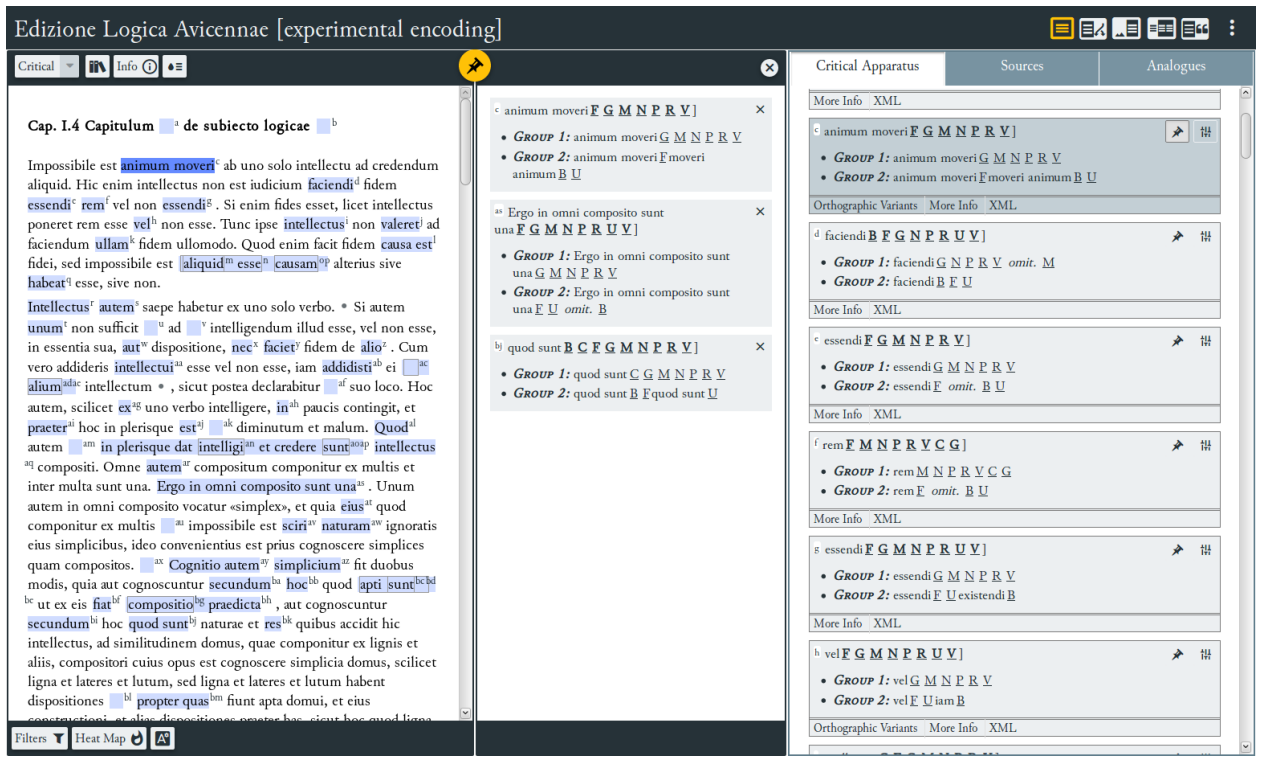

Figure 4. The new features introduced in EVT 2 thanks to the collaboration with the PhiBor project 
At present, EVT 1 is a mature product, but won't receive any further improvement after version 1.3 is released, while EVT 2 is available as a usable beta version and a fully functional release is due at the end of 2019 . The forthcoming release will include all the features present in the XSLTbased EVT 1, i.e. support for diplomatic editions, text-image linking, a sophisticated image viewer with IIIF $^{14}$ support, a powerful text engine, and more. At the same time, the support for critical editions - which was the starting point for EVT2 - will be further improved (such important feature is currently being tested) thanks to the collaboration with other projects and the feedback of many scholars interested in using EVT to publish their editions.

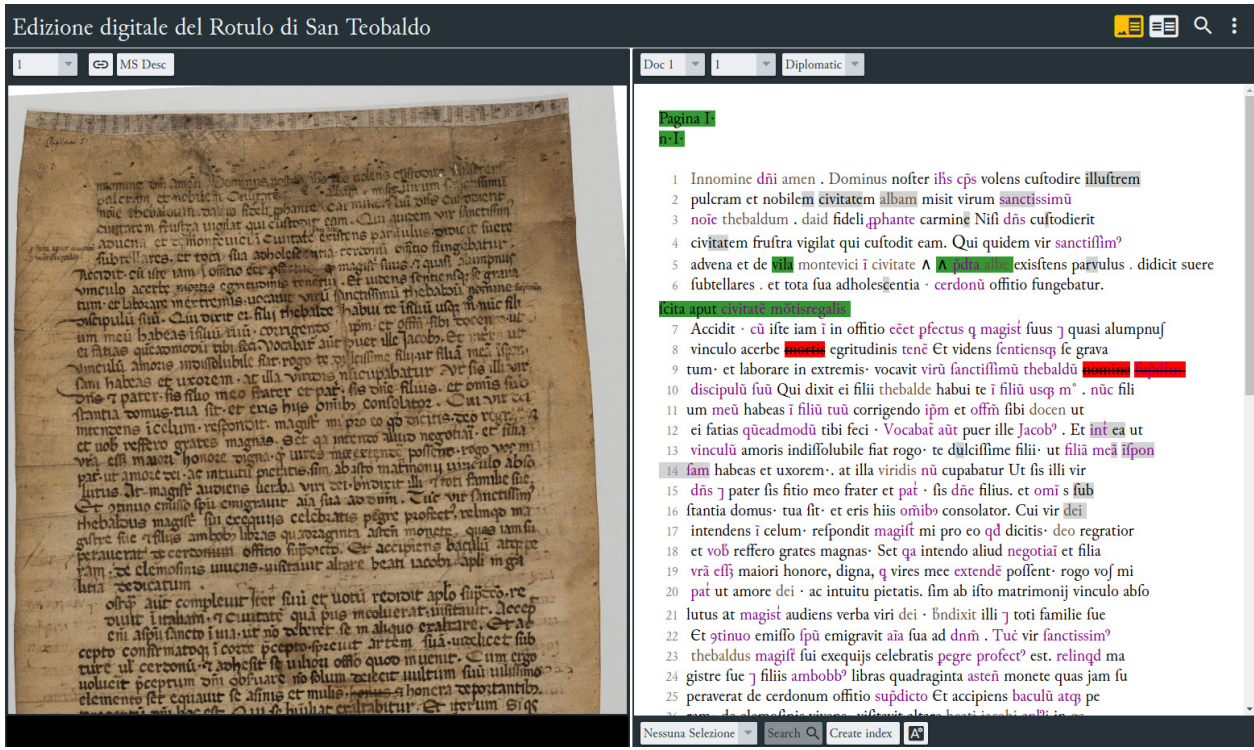

Figure 5. The diplomatic edition features of EVT 1 now available in EVT 2. This screenshot comes from the Vita di San Teobaldo digital edition project, see http:// licodemo.ilc.cnr.it/rotulo-evt/ for a preliminary version.

14. International Image Interoperability Framework, a set of API (Application Programming Interface) specifications with the goal of making it easier to distribute images over the Web. Home page: https://iiif.io/. 
The feature that we deem essential for the next generation of digital editions, however, is the annotation of texts and/or images by the final user: in the current development version there is a collapsible area, called "Pin frame", where the user can collect specific apparatus entries, notes and in the forthcoming stable version - editor's comments, so that they can come back to study them at a later moment. We intend to develop this basic feature into a proper "digital workspace" where users can bookmark such items, add their own annotations related to a specific segment of the text or a detail in a digitized image, and eventually share them with other users. The resulting data will be saved on the edition's server and will represent a progressive enrichment of the same. ${ }^{15}$

Finally, since we chose to only use open Web standards (HTML5, CSS3, Javascript, Angular) we plan to keep this future version of EVT as simple to install, configure and maintain as possible. Thanks to its client-only approach, the current version needs very little, if any, maintenance once the edition has been published on a Web server. Our intent is to design and implement the enhancements described above in such a way as to require the very minimum of resources and to guarantee long term sustainability for EVT-based digital editions.

\section{Theory and practice}

Quelli che s'innamorano della pratica senza la scienza, sono come i nocchieri che entrano in naviglio senza timone o bussola, che mai hanno certezza dove si vadano. Sempre la pratica dev'essere edificata sopra la buona teorica, della quale la prospettiva è guida e porta, e senza questa nulla si fa bene. (Leonardo da Vinci, Trattato della pittura, II.77)

A common feature of many of the first Digital Humanities projects (including digital edition projects) is the fact that they were created to offer a solution to a specific problem, but as they evolved they lead to consider not only other aspects, at first unexplored, of the problem or other related

15. Peter Shillingsburg observes that "Adequate electronic designs for archives and editions will make it feasible and easier to produce new literary criticism that is more broadly based in relevant texts and contexts". Unfortunately "[t]he state of modern electronic archives and editions represents a failure of imagination - a failure to see the problem whole or to recognize the shortfalls of local solutions for local problems" (Shillingsburg 2010, 179, 180-1). 
issues, but also to a broader theoretical reflection. ${ }^{16}$ Practice, one could say, leads to theory, and thence - hopefully — to better practice. As far as EVT is concerned, apart from the technical problems inherent to the development of such a complex application, the great challenges we have had to face are above all two: the general design of the user interface and the search for flexibility with the aim of empowering the editor.

As for the first point, the paradox which presented to us is that of being able to count on an almost infinite storage space, but also that of having to show the multiple layers of edition content (texts, images, notes, etc.) in a two-dimensional space just a little larger than a printed page: the screen of a computer. As long as the DSEs are going to require a computer for their consultation, their use will be that of a research tool and they will not be able to reach the versatility of a traditional printed edition.

The second challenge concerns the creation of a tool flexible enough to take into account, and thus successfully handle, not only the great variability regarding texts and different ecdotic methodologies, but also the complexity and, to a large extent, the redundancy of the XML schemas currently used for the purpose of text encoding. The TEI standard, in fact, often allows us to mark up the same content in many different ways, which creates a serious problem for those who have to foresee how the input texts are encoded in order to process them.

These thoughts on the issues related to the encoding of an edition text led us to a more general reflection, which in part had already occurred at the time of choosing the markup language. Whilst in the traditional workflow of a printed edition all intermediate stages of "processing" (and any possible editorial concepts on the nature of the text) are lost, unless they are made explicit or summarized in the introductory material of the edition itself, the very novelty of digital philology has led many scholars to explain - if not analytically justify — their choices. This is a reflection to which we are "forced" by the fact that in order to use text processing tools we must accept a text representation model which translates into a formal language, the only one that can be processed by a software tool; and this requires that we reflect on what the text is, first of all, but also on the "translation" itself and what it entails (including the risk of being "lost-in-translation").

The limitations of XML, namely the rigidity of the hierarchical structure and the difficulty of representing parallel hierarchies, are due to the nature of this markup language and its design as a tool to solve "computer"

16. This is the theme explored by Elizabeth Burr (2018) in her keynote address at the 2018 EADH conference. 
problems, or, in any case, issues related to a document model that certainly does not include a full DSE. Which is why we chose to base EVT on an open framework, so that it is possible to add further text parsers for non-TEI XML data formats.

The conclusion is that the tools we use are not neutral. On the contrary, they affect us on several different levels. With regard to markup languages, for example, the support for a specific data format determines the choice of processing and visualization tools, and vice versa. This is also a particularly important decision because one of the main objectives of the scholar should be the long-term preservation of the digital edition textual data, which depends precisely on the choices made during the design phase of the edition. Again, separating the edition data from the processing tools is the safest way to ensure its durability in the medium to long term.

The definition of a suitable model for textual data is a critical point with regard to digital philology. If we take into account the various factors that condition the scholar with respect to this fundamental aspect, and assuming that the choice falls on the TEI XML format, we will encounter the following problems:

- first of all, we are forced to have a vision of the text based on the $\mathrm{OHCO}$ concept, ${ }^{17}$ and therefore based on a definition of text as a hierarchical structure;

- secondly, we have the inevitable limitations of XML in implementing this concept: it is certainly a powerful and flexible meta-language, but it still represents a simplification of its predecessor, SGML (Standard Generalized Markup Language, an ISO standard: see http://www.w3.org/MarkUp/SGML/). ${ }^{18}$

- then we have the limitations of the encoding schemas that have been implemented in this language, namely the TEI schemas and Guidelines: not only in terms of incompleteness, which is inevitable and accepted by the TEI editors themselves as it is impossible to pre-

17. Renear 2004. Discussing the shortcomings of this model E. Pierazzo remarked that "The $\mathrm{OHCO}$ model was elaborated by observing the steps and the requirements of the publication process, and not the editorial, scholarly work, so it comes as no surprise to discover that it falls short on the task of providing a viable model for the editorial work" (Pier azzo 2015, 72).

18. An ISO standard, see http://www.w3.org/MarkUp/SGML/ for more information. 
dict every possible need, ${ }^{19}$ but also of redundancy, the possibility of encoding similar phenomena in several different ways; ${ }^{20}$

- finally, each digital edition project defines its own way of using the TEI schemas, which on the one hand helps in limiting redundancy and can make up for incompleteness thanks to the introduction of custom elements, ${ }^{21}$ but it is also an opportunity to introduce nonoptimal solutions, if not actual errors, in the encoding of the edition texts.

Although at the moment it is the most widespread and effective standard, we cannot but conclude that TEI encoding is a non-neutral tool, one which imposes its own modus operandi and constraints on the scholar. In addition to the numerous variables mentioned above regarding philological methods and types of texts to be edited, there is also the fact that inevitably different editors will encode the same text in a different way in spite of sharing the same methodology. 22

This is not all, however, and other factors need to be taken into account:

- a crucial phase is the processing of textual data, the way in which they will be managed by the publication software prior to the next phase, their visualization; the fact that this is a real "black box" should alert on the risks inherent in this step (wrong or under-use of the markup, incorrect or incomplete processing of textual data, inefficiency of tools such as search engines, generation of concordances, collation of witnesses, etc.)

- the visualization of the edition data as a result of their processing is the last phase, perhaps the most critical since for the final user what he sees is, to all intents and purposes, the digital edition; the design of the user interface, the navigation and research tools which are going to be offered to the user, even the use of style-sheets to high-

19. The standard solution of this problem is using generic elements that are adapted to the context, and other types of markup customization.

20. Which is why the TEI editors have published a product, called TEI Simple (https://github.com/TEIC/TEI-Simple), which includes a strict "processing model" making it possible to create digital documents quickly and in a standardized way.

21. Notably, this introduces the risk of losing opportunities for the interchange of encoded textual data.

22. My personal experience, gained from collaborating with several edition projects, leads me to conclude that this is a certainty rather than a possibility. 
light specific textual fragments are all going to have a deep impact on the user experience and on the effectiveness of the edition itself.

While a DSE allows you to go beyond the book form to describe a book, ${ }^{23}$ there is still a lot of theoretical work to be done to identify and accurately describe the limitations imposed by the tools currently available with regard to the modeling, the encoding in a formal language and the subsequent processing and display of the texts of an edition: it is the only way to be able to handle them properly, and to begin to reflect on what could be a better data format than the current one. The proper design and implementation of the user interface for such a tool is essential to effectively browse a DSE and to take full advantage of it. ${ }^{24}$ The degree of complexity of such a tool also determines the work method of a research team: a complex tool will require a team of several people, and probably will also need continuous IT support over time for maintenance and updating, which is essential in the case of any server-side software.

All of this means that developing a software tool such as EVT is not simply complicated from a technical point of view, but it is a process that involves taking into account many crucial philological issues. A somewhat underestimated problem with many digital philology tools is that they have not been designed by philologists, nor have philologists involved in their design and implementation. ${ }^{25}$ Since I am in the fortunate position of being a textual critic who also develops ${ }^{26}$ the tool to be used for the publication

23. "The crucial problem here is simple: the logical structures of the "critical edition" function at the same level as the material being analyzed. As a result, the full power of the logical structures is checked and constrained by being compelled to operate in a bookish format. If the coming of the book vastly increased the spread of knowledge and information, history has slowly revealed the formal limits of all hardcopy's informational and critical powers. The archives are sinking in a white sea of paper" (MCGANN 1997, 21-2).

24. On this subject see Bleier 2018, and in particular Andrews-van Zundert 2018 .

25. To understand the intertwining of such level of technical complexity and of development issues (both having consequences at the "academic" level), and the complexity of its management by humanists, see vAN ZUNDERT 2018.

26. More precisely, I am directing the development of such a tool, which has proved to be a completely new experience, even compared to the DVB project. The traditional research model in the Humanities, that of the scholar working in a completely independent way and presenting the result of his work only at the end of it, is definitely inapplicable in this context, although text encoding is 


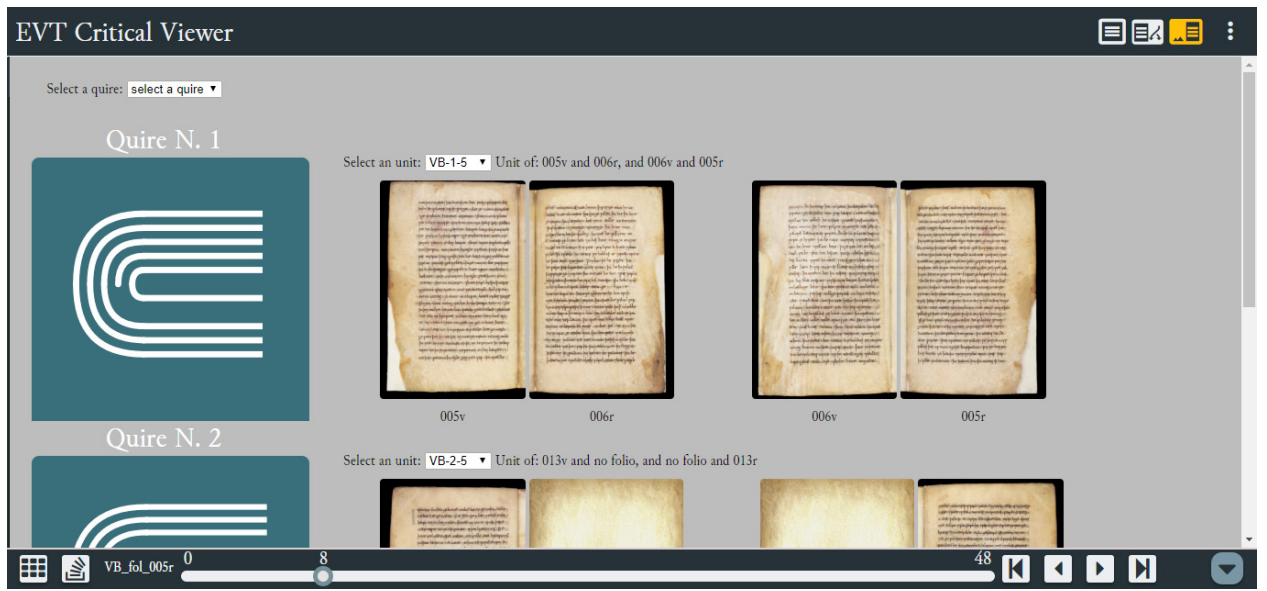

Figure 6. VisColl implementation in EVT 2

of his digital edition projects, the philological issues have always been at the top of the list of priorities, in such a way as to put IT resources at the scholar's service, rather than conditioning the result on the basis of what can be rapidly achieved with the development resources available to a Web developer. From this premise also comes the awareness that such a program must be as versatile and configurable as possible, to satisfy needs that are potentially very different from my own. As mentioned in the previous section, EVT has been designed and developed in such a way as to allow flexibility on several levels:

- the configuration phase allows you to decide the general layout of the UI, which tools to activate and which to leave aside, etc.;

- you can add custom CSS style sheets to change the appearance of the edition text (see above);

equivalent to the traditional philological method, in that it requires the scholar to work autonomously, albeit making use of new technologies. In our work team there is not only a division of tasks such as to make total control of development impossible for a single person, even if he/she is the project director, but there has always been a discussion that allows to confront different ideas and is generally extremely productive. The basic precondition is a clear division of competences and the responsibility of each person for his or her area of competence. 
- you can also add XSLT style sheets to introduce new features independent of the JavaScript parser, e.g. the implementation of VisColl (see Fig. 6); 27

- finally, it is possible to directly modify and improve the current code base.

As a philologist and the first user of this software, however, I do realize that - despite all our precautions - this may not be enough, and that the last point entails such skills as to require the intervention of ICT experts. It is also why we rely so much on feedback from our users, having different points of view and needs (sometimes very different) than those of a single project helps to maintain a broad perspective.

\section{Conclusion}

The factors that can determine the success of digital editions as a comprehensive alternative to traditional editions are only partly of a technical nature: problems such as quotability, evaluation, maintainability, durability and long-term preservation of DSEs belong more to the sphere of politicalinstitutional decisions than to those of implementation by means of IT tools. For example, the longevity of an edition from the point of its persistence on the Web is more related to the institutional decision to offer continued support than to the hardware and software tools (e.g. storage of the edition data in a real cloud network), which are in fact already available and of widespread use for other purposes. From a technical point of view, the separation between data and visualization software is an essential point for the survival of a DSE in the medium to long term. The current standards for graphic and textual data allow for their preservation in the long term, which means that this goal is achievable for the DSE as a whole, provided that a clear data management and — possibly — a data migration strategy is put in place.

What will really make the difference will be a new generation of creation and visualization tools for DSEs that are explicitly designed to meet the needs of textual criticism scholars. To achieve this goal, a close collaboration of scholars is certainly necessary: first at a theoretical level, to discuss and solve problems related to text modeling; secondly, to define

27. VisColl is a software tool to create models of the physical collation of manuscripts, which can subsequently be displayed and studied; home page https:// github.com/leoba/viscoll. 
both the layout of the user interface, and the correct handling of all those textual phenomena whose representation is essential for any critical or diplomatic edition.

Università degli Studi di Torino

\section{Works Cited}

Andrews, Tara L. and Joris J. van Zundert. 2018. "What Are You Trying to Say? The Interface as an Integral Element of Argument". Digital Scholarly Editions as Interfaces, edited by Roman Bleier, Martina Bürgemeister, Helmut W. Klug, Frederike Neuber, and Gerlinde Schneider. Schriften Des Instituts Für Dokumentologie Und Editorik 12: 3-34. Norderstedt: BoD: https://kups.ub.uni-koeln. de/9085/.

Bleier, Roman, Martina Bürgemeister, Helmut W. Klug, Frederike Neuber, and Gerlinde Schneider, eds. 2018. Digital Scholarly Editions as Interfaces. Schriften Des Instituts Für Dokumentologie Und Editorik Vol. 12. Norderstedt: BoD: https:// kups.ub.uni-koeln.de/9085/.

Burr, Elizabeth. 2018. "Why Digital Humanities, and How?" "Data in Digital Humanities” EADH Conference, National University of Ireland, Galway 7-9 December 2018: https://eadh2018eadh.wordpress.com/.

Di Pietro, Chiara. 2016. EVT per le edizioni critiche digitali: progettazione e sviluppo di una nuova GUI basata sullo schema progettuale MVC. MA thesis. Pisa: ETD - Electronic theses and dissertations repository, Università di Pisa.

Di Pietro, Chiara and Roberto Rosselli Del Turco. 2018. "Between Innovation and Conservation: The Narrow Path of User Interface Design for Digital Scholarly Editions". In Bleier, Klug, Neuber, and Schneider. 2018, 133-63: https:// kups.ub.uni-koeln.de/9085/.

Franzini, Greta, Simon Mahony, and Melissa Terras. 2016. A Catalogue of Digital Editions. Digital Scholarly Editing: Theories and Practices, edited by Elena Pierazzo and Matthew J. Driscoll. Cambridge: Open Book Publishers: https:/github.com/ gfranzini/digEds_cat (accessed 18 December 2018).

Garrett, Jesse J. [2005] 2007. “Ajax: A New Approach to Web Applications”. https:// www.semanticscholar.org/paper/Ajax\%3A-A-new-approach-to-web-applicationsGarrett/c440ae765ff19ddd3deda24a92ac39cef9570f1e.

Martignano, Chiara. 2017. Progettazione e sviluppo di un apparato critico modellato sulla tradizione a stampa in EVT. MA thesis. Pisa: ETD - Electronic theses and dissertations repository, Università di Pisa.

McGann, Jerome J. [1995] 1997. "The Rationale of Hypertext”. Electronic Text: Investigations in Method and Theory, edited by Kathryn Sutherland. Oxford: Oxford University Press. Originally published online: http:/www2.iath.virginia.edu/public/ jjm2f/rationale.html (accessed 18 December 2018). 
PhiBor 2014-2019. Philosophy on the Border of Civilizations and Intellectual Endeavours: Towards a Critical Edition of the Metaphysics (Ilāhiyyāt of Kitāb al-Šifä') of Avicenna (Ibn Sinna). ERC Advanced Grant (n. 339621): http://www.avicennaproject. eu/ (accessed 18 December 2018).

Pierazzo, Elena. 2015. Digital Scholarly Editing: Theories, Models and Methods. London: Routledge.

2016. "Quale futuro per le edizioni digitali? Dall'haute couture al prêt-à-porter". Fifth AIUCD Annual Conference: Digital editions: Representation, interoperability, text analysis and infrastructures. Venice, 7-9 September 2016, Università Ca' Foscari: http://www.himeros.eu/aiucd2016/c30.pdf.

Renear, Allen H. 2004. "Text Encoding”. In A Companion to Digital Humanities, edited by Susan Schreibman, Ray Siemens, and John Unsworth. Oxford: Blackwell: http://www.digitalhumanities.org/companion/.

Rosselli Del Turco, Roberto, Giancarlo Buomprisco, Chiara Di Pietro, Julia Kenny, Raffaele Masotti, and Jacopo Pugliese. 2015. "Edition Visualization Technology: A Simple Tool to Visualize TEI-based Digital Editions". Journal of the Text Encoding Initiative 8: 1-21. Selected Papers from the 2013 TEI Conference: https://jtei.revues.org/1077 (accessed 18 December 2018).

Rosselli Del Turco, Roberto, Raffaele Cioffi, and Federica Goria. 2017. The Digital Vercelli Book. A facsimile edition of Vercelli, Biblioteca Capitolare, CXVII, Università di Torino, Collane@unito.it. I http://www.collane.unito.it/oa/items/show/11 (accessed 18 December 2018).

SAhle, Patrick. 2017. A catalog of Digital Scholarly Editions: http://www.digitale-edition.de/ (accessed 18 December 2018).

Salvatori, Enrica, Edilio Riccardini, Laura Balletto, Roberto Rosselli Del Turco, Chiara Alzetta, Chiara Di Pietro, Chiara Mannari, Raffaele Masotti, and Alessio Miaschi, eds. 2014. Codice Pelavicino. Edizione digitale: http://pelavicino.labcd.unipi.it/ (accessed 18 December 2018).

Shillingsburg, Peter. 2010. "How Literary Works Exist: Implied, Represented, and Interpreted". Text and Genre in Reconstruction: Effects of Digitalization on Ideas, Behaviours, Products and Institutions - Open Book Publishers, edited by Willard McCarty, 165-82. Cambridge: Open Book Publishers: https://www.openbookpublishers.com/product/64 (accessed 18 December 2018).

van Zundert, Joris. 2018. "On Not Writing a Review about Mirador: Mirador, IIIF, and the Epistemological Gains of Distributed Digital Scholarly Resources". Digital Medievalist 11 (1): 5: https://journal.digitalmedievalist.org/articles/10.16995/dm.78/ (accessed 18 December 2018). 